\title{
The Design and Performance of a Double Wien Filter Monochromator for Application in TEM
}

\author{
M. Mukai ${ }^{1}$, J. Warner ${ }^{2}$, J.S. Kim ${ }^{2}$, K. Omoto ${ }^{1}$, H. Sawada ${ }^{1}$, A. Kimura ${ }^{1}$, A. Ikeda ${ }^{1}$, J. Zhou $^{1}$, T. \\ Kaneyama $^{1}$ P. D. Nellist ${ }^{2}$ and A. I Kirkland ${ }^{2}$ \\ 1. JEOL Ltd., 1-2 Musashino 3-Chome, Akishima, Tokyo 196, Japan \\ 2. Department of Materials, Parks Road, Oxford OX1 3PH, UK
}

The extensive development of aberration correctors has significantly improved spatial the spatial resolution attainable in both STEM and TEM. In parallel, improved spectrometers have promoted renewed interest in high energy resolution spectroscopy, often at atomic resolution. Both of these developments benefit from quasi monochromatic electron sources for maximum benefit. In the case of TEM imaging these reduce the limiting effects of partial temporal coherence which is of particular importance to imaging at low accelerating voltages and for STEM spectrum imaging they provide improved spectral energy resolution.

This paper describes the design and construction of a monochromator consisting of two dodecapole type Wien-filters with an energy selection slit located between the two filters. A single dodecapole Wien filter is shown in Figure 2 together with the calculated electrostatic and magnetic dipole fields and the electrostatic quadrupole field along the optic axis. In this design each pole acts as both an electrode and as a magnetic pole. Shunts are located at the entrance and exit of the Wien-filter so that the distribution of the dipole electrostatic field can be set equal to the dipole magnetic field both in the inside of the Wien filter and also at the fringing region.

In the design reported here the electron trajectory is parallel at the entrance of the monochromator. The first Wien-filter produces a crossover of the energy-dispersed beam at the slit plane for energy selection. At this plane, the energy dispersion is $22 \mu \mathrm{m} / \mathrm{eV}$ and the width of the energy-selecting slit can be varied from $0.5 \mu \mathrm{m}$ to $9 \mu \mathrm{m}$. The second Wien filter cancels the energy dispersion and the electron trajectory at the exit of monochromator is also parallel. An electrostatic round lens produces a crossover at the exit plane of the monochromator giving a focused achromatic and stigmatic beam. The Wien filters can be turned off whilst maintaining a constant setting of the electrostatic round lens.

Figure 2 shows the intensity profiles of zero-loss peaks recorded on a conventional 4096 x 4096 pixel CCD camera obtained using the minimum width energy selection slit $(0.5 \mu \mathrm{m})$ and 0.1 second acquisition at accelerating voltages of $200 \mathrm{kV}$ and $80 \mathrm{kV}$, respectively. For these two primary energies the FWHM of the zero loss peaks were measured as $36 \mathrm{meV}$ at $200 \mathrm{kV}$ and $26 \mathrm{meV}$ at $80 \mathrm{kV}$ respectively broadening to $72 \mathrm{meV}$ at $200 \mathrm{kV}$ and $56 \mathrm{meV}$ at $80 \mathrm{kV}$ for 100 second acquisitions. Measurement of the detailed profile of the zero loss peak as an indicator for loss spectroscopic performance shows that the zero loss peak $(\mathrm{FWHM}=0.26 \mathrm{eV}$ at $80 \mathrm{kV})$ drops to $1 / 10$ of its initial value at $34 \mathrm{meV}, 1 / 100$ at $112 \mathrm{meV}, 1 / 1000$ at $227 \mathrm{meV}, 1 / 10000$ at $350 \mathrm{meV}$.

In conclusion a field emission gun with a double Wien filter monochromator has been developed for operation at 80 and $200 \mathrm{kV}$ achieving ultimate energy resolutions (measured as the FWHM of zero-loss peaks) were measured of $36 \mathrm{meV}$ at $200 \mathrm{kV}$ and $26 \mathrm{meV}$ at $80 \mathrm{kV}$. This paper will highlight recent low voltage imaging of defects and dislocations in graphene obtained with this instrument. 
References:

[1] M. Mukai et al.: Microscopy \& Microanalysis, 13 (Supple.2) (2007), p. 1242

[2] Y. Sato et al, Ultramicroscopy, 111, (2011), p. 1381.

[3] G. Martinez, K. Tsuno: Ultramicroscopy 93 (2002), p. 253.

[4] K. Tsuno, D. Ioanoviciu and G. Martinez: J. Microsc. 217 (2005), p. 205.

[5] The authors acknowledge financial support from EPSRC and the EU.

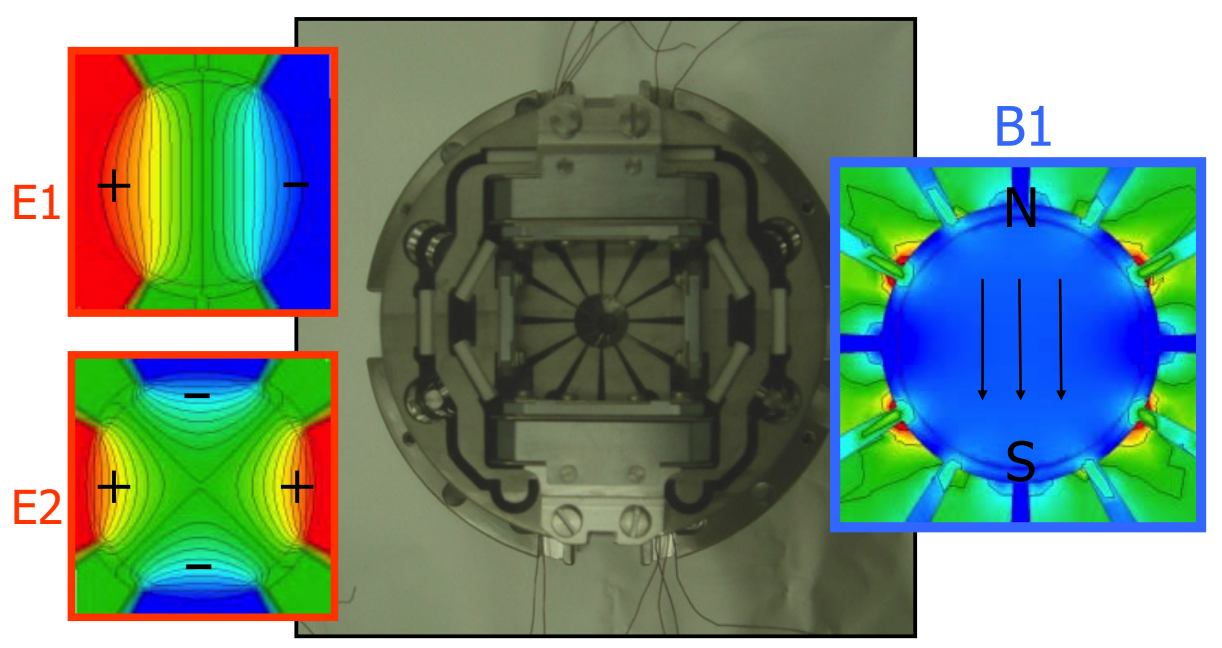

Figure 1. Top view of the dodecapole type Wien-filter and the calculated dipole electric field (E1), dipole magnetic field (B1) and quadrupole electric field (E2).
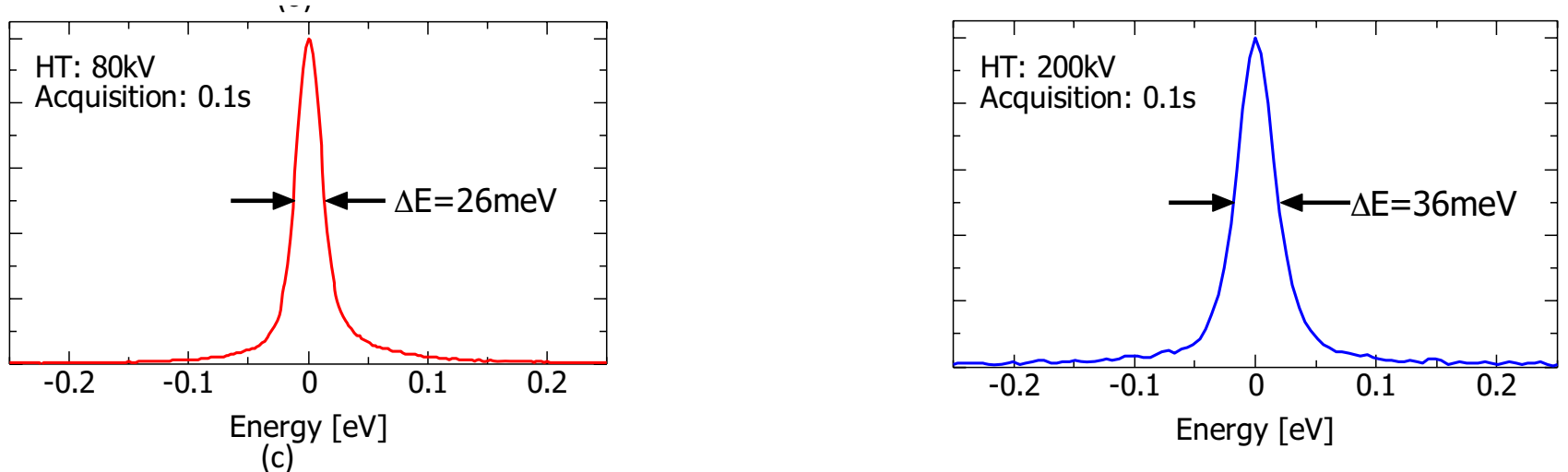

Figure 2. Intensity profiles of zero-loss peaks obtained at $80 \mathrm{kV}$ and $200 \mathrm{kV}$ primary energy with a $0.1 \mathrm{~s}$ acquisition on a 4096 x 4096 pixel CCD detector. 